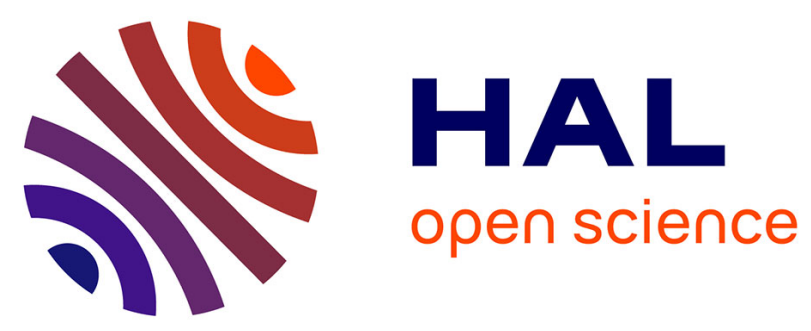

\title{
Supervision of Nonlinear Networked Control Systems Under Network Constraints
}

Afef Najjar, Thach Ngoc Dinh, Messaoud Amairi, Tarek Raïssi

\section{To cite this version:}

Afef Najjar, Thach Ngoc Dinh, Messaoud Amairi, Tarek Raïssi. Supervision of Nonlinear Networked Control Systems Under Network Constraints. 2019 4th Conference on Control and Fault Tolerant Systems (SysTol), Sep 2019, Casablanca, Morocco. pp.270-275, 10.1109/SYSTOL.2019.8864767. hal-02434061

\section{HAL Id: hal-02434061 https://hal-cnam.archives-ouvertes.fr/hal-02434061}

Submitted on 19 Jan 2020

HAL is a multi-disciplinary open access archive for the deposit and dissemination of scientific research documents, whether they are published or not. The documents may come from teaching and research institutions in France or abroad, or from public or private research centers.
L'archive ouverte pluridisciplinaire HAL, est destinée au dépôt et à la diffusion de documents scientifiques de niveau recherche, publiés ou non, émanant des établissements d'enseignement et de recherche français ou étrangers, des laboratoires publics ou privés. 


\title{
Supervision of Nonlinear Networked Control Systems Under Network Constraints
}

\author{
Afef Najjar ${ }^{1}$, Thach Ngoc Dinh ${ }^{2}$, Messaoud Amairi ${ }^{1}$, Tarek Raïssi ${ }^{2}$
}

\begin{abstract}
The remote supervision for a class of nonlinear systems in the presence of additive disturbances and measurement noises is considered in this paper. The communication network may introduce time delays while exchanging data among sites connected to the network medium (i.e., the data acquisition site and the remote plant site). Two different approaches are presented in this paper. The first one uses a conventional estimator-based predictor when the uncertainties are supposed to be known. In the case of unknown but bounded uncertainties by known bounds, an interval estimation-based predictor evaluating the set of admissible values for the state is investigated. The state prediction techniques are used to compensate the effect of network-induced delays. Simulation results are introduced to illustrate the efficiency of the proposed techniques.
\end{abstract}

Index Terms-Nonlinear networked control system (NNCS), time-varying delay, state estimation, interval observer, predictor.

\section{INTRODUCTION}

Recently, networked control system (NCS) has gone through wide studies due to its many advantages, such as reduced wiring cost, flexible maintenance and high reliability [1]-[3]. NCS has been found in many applications including manufacturing plants, automobiles, aircraft and remote surgery [4]. In general, a NCS consists of sensors, actuators and controllers whose operations are distributed at different geographical locations and coordinated via information exchanged over shared communication networks [4]. Using a shared band limited digital network as medium to connect could cause some imperfections such as network-induced time delay, data packet dropout, quantization, sampling and data rate constraints [5]-[7]. All these imperfections can introduce instability and performance degradation which imply that the stability analysis and control design become more difficult to be investigated.

In the literature, many methods have been proposed to deal with performance degradation induced by communication networks. For example, NCS was considered as a switched linear system in many works, see for instance [8]-[10]. In [9], the system was presented as a discrete-time switched linear uncertain system subject to time-varying delay, variable transmission interval and communication

\footnotetext{
1 Afef Najjar and Messaoud Amairi are with University of Gabes, ENIG, Research Labo MACS LR16ES22, Gabes 6029, Tunisia afef.najjardenig.rnu.tn amairi.messaoudeieee.org

2 Thach Ngoc Dinh and Tarek Raïssi are with Conservatoire National des Arts et Métiers (CNAM), Cedric - Laetitia, 292 Rue St-Martin, 75141 Paris Cedex 03, France. ngoc-thach. dinh@lecnam.net tarek.raissi@cnam.fr
}

constraints. A finite number of Linear Matrix Inequalities (LMI) was used to analyze the system stability. Similarly in [11] and [12], the NCS has been presented in the discretetime domain in the presence of both network delays and data dropout which were modeled as Bernoulli event in [11] and as two Markov chains in the action and measurement channels in [12]. On the other hand, several alternative solutions have been proposed to achieve stability conditions of NCS. In [13] and [14], a robust $H_{\infty}$ controller has been designed to cope with the network-induced delay and/or packet loss. Also, a fuzzy proportional integral derivative controller (PID) was used to overcome the problem of random network delay [15] and a semi-global practical stability of the closed-loop system was given in term of gains in [5].

In addition, the supervision problem is very important in industry. Many solutions already exist for linear systems and a number of nonlinear structures. For the latter situation, a state prediction, compensating large network-induced delays, is considered in this paper. State estimation over networks is important in applications such as remote sensing, space exploration, and sensor networks. However in real-life applications, the knowledge of full states of a NCS is rather complicated [16]. To solve this issue, a natural approach is to construct a state estimator from the available data. In [17], [18], an observer-based control for NCS has been mentioned. In [17], a switched fuzzy state observer was designed despite random measurements and time delays while authors in [18] have proposed an observer-based residual generator dealing with a robust fault detection filter (FDF) design and optimization problem.

In the presence of unknown additive disturbances or/and measurement noises (i.e., unknown uncertainties), like the case of the considered NNCS architecture, all these conventional observers converging to the ideal value of the state cannot be realized. Nevertheless, an interval estimation providing guaranteed upper and lower bounds for the state still remain feasible: an interval observer can compute robustly the set of all admissible values instead of a single point approximation. Roughly speaking, interval observer is composed of two classical ones allowing to determine two bounds which enclose the state under the assumptions that the uncertainties are unknown but bounded by known bounds. That is why interval observers have been becoming more and more popular in the last few years [19]-[23].

In the present work, we deal with a supervision strategy for a class of Nonlinear Networked Control Systems 
(NNCS) subject to uncertain network-induced delays which are assumed to be unknown but bounded with an upper bound and a lower bound. Different from many researches [24], [25], we treat the case of large network-induced delays. In other words, we allow the upper bound of these delays to be greater than the sampling period. Also, additive disturbances and measurement noises are considered in this NNCS structure. In the absence of unknown uncertainties (i.e., disturbances and measurement noises) and after a finite time depending on the upper bound of network-induced delays, the conventional estimator-based predictor can be achieved. Next, after a finite time depending on the upper bound of network-induced delays, the interval estimationbased predictor provides upper and lower bounds for each component of the solutions when unknown but bounded uncertainties are present. Hence, the current state of the remote plant is reconstructed thanks to the state predictor outputs in both approaches.

The rest of the paper is organized as follows. Section II is formulated to describe the studied system. The classical approach is investigated in Section III. Section IV develops the interval approach. The effectiveness of the proposed supervision schematic is drawn in Section $\mathrm{V}$ via a numerical example. Finally, Section VI gives a brief conclusion.

Notations and definitions: $\mathbb{R}$ and $\mathbb{N}$ refer to the sets of real and natural numbers, respectively. Denote the set of eigenvalues of the matrix $A \in \mathbb{R}^{n \times n}$ by $\lambda(A)$ and $\operatorname{Re}(z)$ is the real part of the complex number $z$. $\mathbb{H}$ is the set of Hurwitz matrices from the set $\mathbb{R}^{n \times n}$, i.e. $R \in \mathbb{H} \Leftrightarrow \operatorname{Re}(\lambda)<0, \quad \forall \lambda \in \lambda(R)$. $\mathbb{M}$ denotes the set of Metzler matrices from the set $\mathbb{R}^{n \times n}$, i.e. $R=\left\{r_{i j}\right\}_{i, j=1}^{n} \in \mathbb{M} \Leftrightarrow r_{i, j} \geq 0$ for $i \neq j$. For a variable $x$, the upper and lower bounds are denoted by $\bar{x}$ and $\underline{x}$ respectively, such that $\underline{x} \leq x \leq \bar{x}$ and the relation $\leq$ is interpreted elementwise for vectors and matrices. Given a matrix $R \in \mathbb{R}^{n \times m}$, denote $R^{+}=\max \{0, R\}$ and $R^{-}=R^{+}-R$. The matrix of absolute values of all elements of a matrix $M \in \mathbb{R}^{n \times m}$ is $|M|=M^{+}+M^{-} . E_{p} \in \mathbb{R}^{p}$ is stated for a vector with unit elements and $I_{n}$ denotes the identity matrix of $n \times n$ dimension. Let $\left\{t_{k}, k \in \mathbb{N}\right\}$ be the sequence of sampling instants such that $t_{k+1}-t_{k}=T$ and $\lim _{k \rightarrow \infty} t_{k}=\infty, T$ is the sampling period and $t_{k}=k T$.

\section{SYSTEM DESCRIPTION AND PROBLEM STATEMENT}

In this paper, a supervisory approach is developed to allow users to send and receive data from local and remote sites given them the impression that they control the plant directly and this latter is fully transparent for them. We focus here on the NNCS whose the general architecture is depicted in Fig. 1. We have two distant sites: the data acquisition site and the remote plant site connected by the network. The first site is charged with supervising and controlling the remote physical plant. Before being transmitted by the communication medium, all data should be passed by a sampling stage which is done with a constant period $T$ in our case. The network introduces a delay $d(t)$. This delay is assumed to be unknown but upper bounded by a known value $\bar{d}$ as follows

$$
0 \leq d(t) \leq \bar{d}, \quad \forall t
$$

The considered physical plant of this NNCS is nonlinear

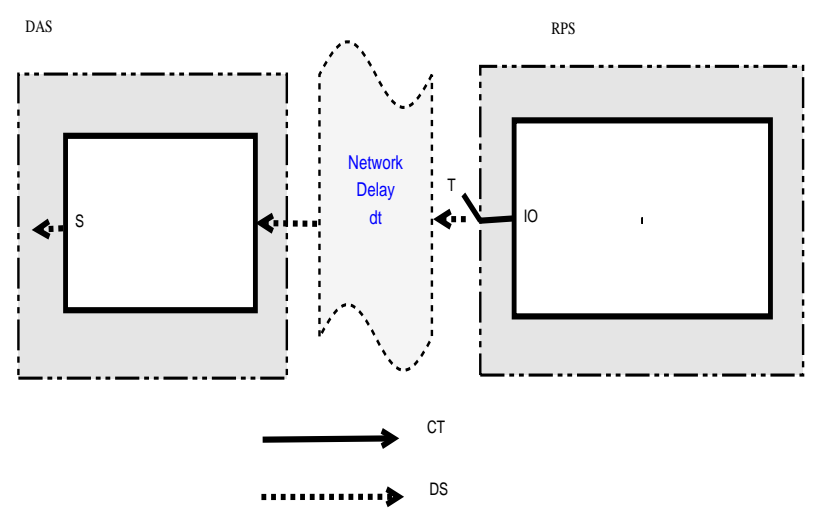

Fig. 1: The general NNCS schematic.

and described by the following differential equation:

$$
\left\{\begin{array}{l}
\dot{x}(t)=A x(t)+F(u(t), y(t))+w(t), \\
y(t)=C x(t)+v(t),
\end{array}\right.
$$

where $x \in \mathbb{R}^{n}$ is the process state, $u \in \mathbb{R}^{m}$ is the vector of inputs, $y \in \mathbb{R}^{p}$ is the output, $w \in \mathbb{R}^{n}$ and $v \in \mathbb{R}^{p}$ are the disturbances and the measurement noises, respectively. $F(u(t), y(t))$ is a nonlinear function. The matrices $A$ and $C$ are time-invariant matrices with proper dimensions.

The objective of this note is to supervise remotely the nonlinear system (2) corrupted by uncertainties, through network introducing delay $d(t)$.

Throughout this work, the next assumptions are made.

Assumption 1: The communication channel is noiseless, and there are no packet dropouts.

Assumption 2: The pair $(A, C)$ is detectable.

Due to the fact that all data should be sampled before being transmitted over the network, the next assumptions are related to the sampling time.

Assumption 3: The sampling network-induced delay $d\left(t_{k}\right)$ is unknown but upper bounded with a known bound as in (1) and its upper bound $\bar{d}$ is a multiple of the sampling period $T$.

Assumption 4: For any sampling instant of time $t_{k} \geq \bar{d}$, the information on the control signal $u\left(t_{k}\right)$, the information on the outputs $y\left(t_{k}\right)$ in the time interval $\left[t_{k}-\bar{d}, t_{k}\right)$ can be stored and used.

Discussions of the assumptions: The Assumption 1 is used for the sake of simplicity. Indeed, all the results in the next sections can be extended to the case where there are packet dropouts. Assumption 2 is necessary for state estimation in the remote plant site and means also that there exists a constant matrix $L$ such that $A-L C$ is Hurwitz. Assumption 3 is is related to the real case and can be frequently satisfied in practice. Assumption 4 is needed for the predictor design. 


\section{ClASSICAL APPROACH}

This section provides converging estimation of the unavailable plant states using the Luenberger observer. However, it can be applied only when the functions $w(t)$ and $v(t)$ are known. As shown in Fig. 2, the NNCS schematic is composed of two sites: the data acquisition site and the remote process site. The first consists of the discrete state predictor while the second contains the continuous physical process, actuator, sensor, continuous local Luenberger observer and Input Set Point Generation (I.S.P.G), where the plant, actuator and sensor are compounded.

The scenario is as follows. The input set point generation

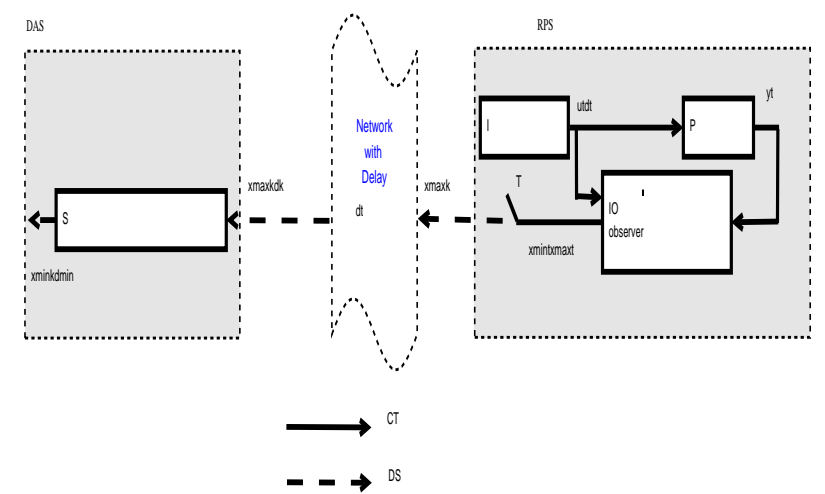

Fig. 2: The classical estimator-based predictor schematic.

calculates and sends the continuous signal $u(t)$ to the plant, periodically. Processing the input $u(t)$, the physical plant returns an output $y(t)$ which will be used by the Luenberger observer with the input to estimate the process state $\hat{x}(t)$. Then, $\hat{x}(t)$ is sampled with the constant sampling period $T$ and it is transmitted to the data acquisition site via the communication medium. The predictor receives the upperdelayed observed state $\hat{z}\left(\frac{t_{k}}{T}-\frac{\bar{d}}{T}\right)$, and regenerates the sampling estimated state $\hat{z}\left(\frac{t_{k}}{T}\right)$ after a finite time $t_{k}=\bar{d}$.

Remark 1: It is worth noting that the network-induced delay is unknown, thus in the Data Acquisition Site we have no access at $\frac{t_{k}}{T}-\frac{d}{T}$. The problem becomes more challenging compared with knowing $d(t)$. As a solution, from the output data received at the upper bound of delay $\frac{t_{k}}{T}-\frac{\bar{d}}{T}$, we use $\hat{z}\left(\frac{t_{k}}{T}-\frac{\bar{d}}{T}\right)$ to reconstruct $\hat{z}\left(\frac{t_{k}}{T}\right)$ after a finite time $t_{k}=\bar{d}$.

Now, let's introduce the Luenberger observer in the Remote Plant Site.

If Assumption 2 holds, i.e., there exists a matrix $L \in \mathbb{R}^{n \times p}$ such that $A-L C \in \mathbb{H}$, then the system

$$
\hat{x}(t)=A \hat{x}(t)+F(u(t), y(t))+L(y(t)-C \hat{x}(t))+w(t)-L v(t)
$$

is an asymptotic observer for (2), i.e., $\lim _{t \rightarrow \infty}|x(t)-\hat{x}(t)|=0$.

Assumption 5: For any sampling instant of time $t_{k} \geq \bar{d}$, the information on the estimated state $\hat{z}\left(t_{k}\right)$ in the time interval $\left[t_{k}-\bar{d}, t_{k}\right)$ can be stored and used.

Theorem 1: Let Assumptions 1-5 hold, for all $t_{k} \geq \bar{d}$, the predictor for (2) is given by

$$
\begin{aligned}
\hat{z}(k) & =\Phi^{k_{1}} \hat{z}\left(k-k_{1}\right) \\
& +\sum_{j=1}^{k_{1}} \Phi^{k_{1}-j} T F\left(u\left(k-k_{1}+j-1\right), y\left(k-k_{1}+j-1\right)\right) \\
& +\sum_{j=1}^{k_{1}} \Phi^{k_{1}-j} L_{1}\left(y\left(k-k_{1}+j-1\right)-C \hat{z}\left(k-k_{1}+j-1\right)\right), \\
& +\sum_{j=1}^{k_{1}} \Phi^{k_{1}-j} T w\left(k-k_{1}\right)+\sum_{j=1}^{k_{1}} \Phi^{k_{1}-j} T L_{1} v\left(k-k_{1}\right)
\end{aligned}
$$

where $j=1,2,3, \ldots, k_{1}, k=\frac{t_{k}}{T}, k_{1}=\frac{\bar{d}}{T}, \Phi=\left(I_{n}+T A\right)$ and $L_{1}=T L$.

Proof 1: It is important to mention that the estimated state $\hat{x}(t)$ should be sampled before being transmitted via communication network, the state observer (3) is represented under discrete-time form through Euler approximation with the sampling period $T$ as follows:

$$
\begin{aligned}
\hat{z}(k+1) & =\Phi \hat{z}(k)+T F(u(k), y(k))+L_{1}(y(k)-C \hat{z}(k)) \\
& +T w(k)-L_{1} v(k),
\end{aligned}
$$

where $\Phi=\left(I_{n}+T A\right), L_{1}=T L$.

From (5) and based on the output data received at $k-k_{1}$, the state predictions from $k-k_{1}+1$ to $k$ are calculated successively as:

$$
\begin{aligned}
\hat{z}\left(k-k_{1}+1\right) & =\Phi \hat{z}\left(k-k_{1}\right)+T F\left(u\left(k-k_{1}\right), y\left(k-k_{1}\right)\right) \\
+ & L_{1}\left(y\left(k-k_{1}\right)-C \hat{z}\left(k-k_{1}\right)\right) \\
+ & T w\left(k-k_{1}\right)-L_{1} v\left(k-k_{1}\right) \\
\hat{z}\left(k-k_{1}+2\right) & =\Phi \hat{z}\left(k-k_{1}+1\right) \\
& +T F\left(u\left(k-k_{1}+1\right), y\left(k-k_{1}+1\right)\right) \\
& +L_{1}\left(y\left(k-k_{1}+1\right)-C \hat{z}\left(k-k_{1}+1\right)\right) \\
& +T w\left(k-k_{1}+1\right)-L_{1} v\left(k-k_{1}+1\right)
\end{aligned}
$$

$$
\begin{aligned}
\hat{z}(k) & =\Phi \hat{z}(k-1)+T F(u(k-1), y(k-1)) \\
& +L_{1}(y(k-1)-C \hat{z}(k-1))+T w(k-1)-L_{1} v(k-1)
\end{aligned}
$$

Combining all these equalities, we can conclude that the formula (4) is a predictor for the plant (2).

\section{INTERVAL APPROACH}

In this part, an interval approach is investigated. Different from the classical one, interval observer computes the guaranteed set of admissible values (interval). Theorem 1 gives in finite time a prediction of any solution $x(t)$ of (2). However, the prediction cannot be used when the disturbances $w$ and $v$ are unknown. The second objective of the present note is to overcome this limitation by assuming only that the bounds $\bar{w}, \underline{w}$ and $\bar{v}, \underline{v}$ are known.

Assumption 6: The measurement noises and the additive disturbances are unknown but bounded with known bounds:

$$
\underline{w} \leq w(t) \leq \bar{w}, \quad|v(t)| \leq \bar{V} E_{p}, \quad \forall t \geq 0
$$


where $\bar{w}, \underline{w} \in \mathbb{R}^{n}$ and $\bar{V} \in \mathbb{R}_{+}$.

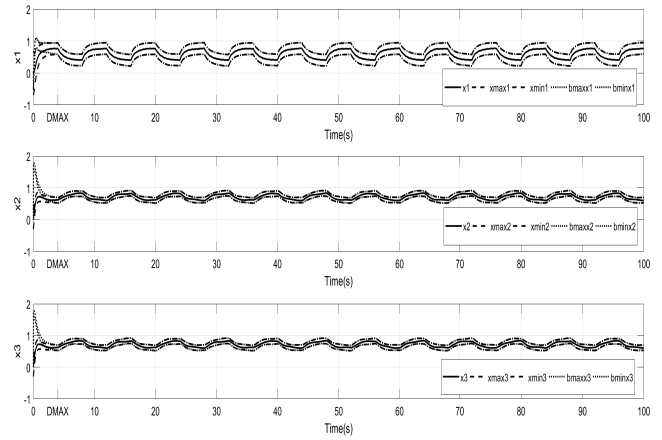

Fig. 3: The interval estimator-based predictor schematic.

As shown in Fig. 3, the scenario is as follows. Periodically, the input set point generation calculates and sends the continuous signal $u(t)$ to the plant. Processing the input $u(t)$, the physical plant returns an output $y(t)$ which is used by the interval estimator with the input to give the admissible bounds $\bar{x}(t)$ and $\underline{x}(t)$ of the state. Then, $\bar{x}(t)$ and $\underline{x}(t)$ are sampled with the constant sampling period $T$. The predictor receives the upper-delayed deviations $\bar{z}\left(\frac{t_{k}}{T}-\frac{\bar{d}}{T}\right), \underline{z}\left(\frac{t_{k}}{T}-\frac{\bar{d}}{T}\right)$ and regenerates the sampling upper and lower bounds of the state $\bar{z}\left(\frac{t_{k}}{T}\right)$ and $\underline{z}\left(\frac{t_{k}}{T}\right)$ after a finite time $t_{k}=\bar{d}$.

Remark 2: As the same problem in the conventional approach, the network-induced delay $d(t)$ is unknown. Thus from the output data received at the upper bound of delay $\frac{t_{k}}{T}-\frac{\bar{d}}{T}$, we use $\bar{z}\left(\frac{t_{k}}{T}-\frac{\bar{d}}{T}\right), \underline{z}\left(\frac{t_{k}}{T}-\frac{\bar{d}}{T}\right)$ to reconstruct $\bar{z}\left(\frac{t_{k}}{T}\right), \underline{z}\left(\frac{t_{k}}{T}\right)$ after a finite time $t_{k}=\bar{d}$.

Now, let's introduce the interval observer in the Remote Plant Site. Generally, it is not possible to compute a gain $L$ satisfying $(A-L C) \in \mathbb{H} \cap \mathbb{M}$. Therefore, a change of coordinates with a nonsingular matrix can be a solution of this restriction.

It is established that if Assumptions 2 and 6 hold and if there exists a change of coordinates $S$ such that $S(A-L C) S^{-1} \in$ $\mathbb{H} \cap \mathbb{M}$, then the system

$$
\begin{aligned}
\dot{\hat{x}}^{+}(t) & =\tilde{A} \hat{x}^{+}(t)+S F(u(t), y(t))+\tilde{L}\left(y(t)-\tilde{C} \hat{x}^{+}(t)\right) \\
& +S^{+} \bar{w}-S^{-} \underline{w}+|\tilde{L}| E_{p} \bar{V} \\
\dot{\hat{x}}^{-}(t) & =\tilde{A} \hat{x}^{-}(t)+S F(u(t), y(t))+\tilde{L}\left(y(t)-\tilde{C} \hat{x}^{-}(t)\right) \\
& +S^{+} \underline{w}-S^{-} \bar{w}-|\tilde{L}| E_{p} \bar{V}
\end{aligned}
$$

where $\tilde{A}=S A S^{-1}, \tilde{L}=S L, \tilde{C}=C S^{-1}$, associated with the initial conditions

$$
\dot{\hat{x}}^{+}(0)=S^{+} \bar{x}_{0}-S^{-} \underline{x}_{0}, \quad \dot{\hat{x}}^{-}(0)=S^{+} \underline{x}_{0}-S^{-} \bar{x}_{0},
$$

and the bounds of the solution $x(t)$

$$
\begin{aligned}
\bar{x}(t) & =S^{+} \hat{x}^{+}(t)-S^{-} \hat{x}^{-}(t) \\
\underline{x}(t) & =S^{+} \hat{x}^{-}(t)-S^{-} \hat{x}^{+}(t)
\end{aligned}
$$

is an interval observer [26] for the system (2), i.e.,

$$
\underline{x}(t) \leq x(t) \leq \bar{x}(t) .
$$

Assumption 7: For any sampling instant of time $t_{k} \geq \bar{d}$, the information on the sampling upper and lower bounds $\underline{z}\left(t_{k}\right)$, $\bar{z}\left(t_{k}\right)$ in the time interval $\left[t_{k}-\bar{d}, t_{k}\right)$ can be stored and used.

Theorem 2: Let Assumptions (1)-(4) and (6)-(7) hold, for all $t_{k} \geq \bar{d}$, then the equations

$$
\begin{aligned}
\hat{z}^{+}(k) & =\Phi^{k_{1}} \hat{z}^{+}\left(k-k_{1}\right) \\
& +\sum_{j=1}^{k_{1}} \Phi^{k_{1}-j} T S F\left(u\left(k-k_{1}+j-1\right), y\left(k-k_{1}+j-1\right)\right) \\
& +\sum_{j=1}^{k_{1}} \Phi^{k_{1}-j} \tilde{L_{1}}\left(y\left(k-k_{1}+j-1\right)-\tilde{C} \hat{z}^{+}\left(k-k_{1}+j-1\right)\right) \\
& +\sum_{j=1}^{k_{1}} \Phi^{k_{1}-j} \bar{\beta}, \\
\hat{z}^{-}(k) & =\Phi^{k_{1}} \hat{z}^{-}\left(k-k_{1}\right) \\
& +\sum_{j=1}^{k_{1}} \Phi^{k_{1}-j} T S F\left(u\left(k-k_{1}+j-1\right), y\left(k-k_{1}+j-1\right)\right) \\
& +\sum_{j=1}^{k_{1}} \Phi^{k_{1}-j} \tilde{L_{1}}\left(y\left(k-k_{1}+j-1\right)-\tilde{C} \hat{z}^{-}\left(k-k_{1}+j-1\right)\right) \\
& +\sum_{j=1}^{k_{1}} \Phi^{k_{1}-j} \underline{\beta},
\end{aligned}
$$

with $j=1,2,3, \ldots, k_{1}, k=\frac{t_{k}}{T}, k_{1}=\frac{\bar{d}}{T}, \tilde{L_{1}}=T \tilde{L}$ and

$$
\begin{aligned}
& \bar{z}(k)=S^{+} \hat{z}^{+}(k)-S^{-} \hat{z}^{-}(k) \\
& \underline{z}(k)=S^{+} \hat{z}^{-}(k)-S^{-} \hat{z}^{+}(k)
\end{aligned}
$$

are the predictor for (2).

Proof 2: Due that all data should be sampled before being transmitted via the communication network, the interval observer (7) is represented under discrete-time form through Euler approximation with the sampling period $T$ as follows:

$$
\begin{aligned}
\hat{z}^{+}(k+1) & =\Phi \hat{z}^{+}(k)+T S F(u(k), y(k)) \\
& +\tilde{L_{1}}\left(y(k)-\tilde{C} \hat{z}^{+}(k)\right)+\bar{\beta}, \\
\hat{z}^{-}(k+1) & =\Phi \hat{z}^{-}(k)+T S F(u(k), y(k)) \\
& +\tilde{L_{1}}\left(y(k)-\tilde{C} \hat{z}^{-}(k)\right)+\underline{\beta},
\end{aligned}
$$

where $\Phi=\left(I_{n}+T \tilde{A}\right), \tilde{L_{1}}=T \tilde{L}, \bar{\beta}=T\left(S^{+} \bar{w}-S^{-} \underline{w}+|\tilde{L}| E_{p} \bar{V}\right)$ and $\beta=T\left(S^{+} \underline{w}-S^{-} \bar{w}-|\tilde{L}| E_{p} \bar{V}\right)$. From (14) and based on the output data received at $k-k_{1}$, the state prediction from $k-k_{1}+1$ to $k$ are calculated successively as:

$$
\begin{aligned}
\hat{z}^{+}\left(k-k_{1}+1\right) & =\Phi \hat{z}^{+}\left(k-k_{1}\right)+T S F\left(u\left(k-k_{1}\right), y\left(k-k_{1}\right)\right) \\
& +\tilde{L_{1}}\left(y\left(k-k_{1}\right)-\tilde{C} \tilde{z}^{+}\left(k-k_{1}\right)\right)+\bar{\beta}
\end{aligned}
$$




$$
\begin{aligned}
& \hat{z}^{+}\left(k-k_{1}+2\right)=\Phi \hat{z}^{+}\left(k-k_{1}+1\right) \\
& +\operatorname{TSF}\left(u\left(k-k_{1}+1\right), y\left(k-k_{1}+1\right)\right) \\
& +\tilde{L_{1}}\left(y\left(k-k_{1}\right)-\tilde{C} \hat{z}^{+}\left(k-k_{1}\right)\right)+\bar{\beta} \\
& \begin{aligned}
\hat{z}^{+}(k) & =\Phi \hat{z}^{+}(k-1)+\operatorname{TSF}(u(k-1), y(k-1)) \\
& +\tilde{L_{1}}\left(y(k-1)-\tilde{C} \hat{z}^{+}(k-1)\right)+\bar{\beta}
\end{aligned}
\end{aligned}
$$

Combining all these equalities, we have (11). Similarly, one can compute the lower prediction $\hat{z}^{-}(k)$ as in (12). Hence, taking into account the change of coordinates $S$, we can conclude that the obtained system is a predictor for the physical plant (2).

\section{Simulations Results}

In order to show the efficiency of our remote supervision schematic, we consider a nonlinear system of the form (2) with $A=\left(\begin{array}{ccc}-1 & 0.1 & 1 \\ 0.02 & -1.2 & 1 \\ 0.02 & 0 & -3\end{array}\right)$, $F(u(t), y(t))=\left(\begin{array}{c}\sin (u(t) y(t)) \\ 2 \cos (y(t)) \\ \sin (y(t))\end{array}\right), C=\left(\begin{array}{lll}1 & 1 & 0\end{array}\right)$, $w(t)=\left[\begin{array}{lll}0.04 \sin (10 t) & 0.04 \sin (2 t) & 0.04 \cos (20 t)\end{array}\right]^{T}$, $\bar{w}=\left[\begin{array}{lll}0.04 & 0.04 & 0.04\end{array}\right]^{T}, \underline{w}=-\bar{w}$ and $v(t)=0.02 \cos (50 t)$ with $\bar{V}=0.02$.

The network-induced delay is given by $d(t)=1+\cos (2 t)$ which is bounded by $\bar{d}=2 s$. The I.S.P.G generates an input signal $u(t)=0.5 \sin (t)+0.1 \cos (t)$.

\section{A. Classical approach}

To estimate the plant states, Luenberger observer is designed as (3) with the gain $L=$ $\left[\begin{array}{lll}0.0092 & 0.0000 & -0.001\end{array}\right]^{T}$.

Under the sampling period $\mathrm{T}=0.01 \mathrm{~s}$, system (3) is sampled and we obtain (5) with $\Phi=\left(\begin{array}{ccc}0.9900 & 0.0010 & 0.0100 \\ 0.0002 & 0.9880 & 0.0100 \\ 0.0002 & 0 & 0.9700\end{array}\right)$ and $L_{1}=\left[\begin{array}{lll}0.0092 & 0.0000 & -0.001\end{array}\right]^{T}$.

The simulation results are plotted in Fig. 4. Both Luenberger observer $\hat{x}$ in (3) and the predictor $\hat{z}$ in (5) are plotted. After a finite time $\bar{d}=2 s$, the predictor converges to the real state, that confirms our theoretical developments.

\section{B. Interval approach}

In this section, we compute a matrix $L$ such that $A-L C \in$ $\mathbb{H}$. We find $L=\left[\begin{array}{lll}0.0092 & 0.0000 & -0.001\end{array}\right]^{T}$. However, the matrix $A-L C$ is Hurwitz but it is not Metzler. Thus, a transformation of coordinates is needed as explained in

Section IV. we can verify that

$$
\begin{aligned}
S(A-L C) S^{-1} & =\left(\begin{array}{ccc}
-1.2399 & 0.0000 & 0.0000 \\
0.1000 & -1.8371 & 0.0000 \\
0.2000 & 0.3000 & -3.0456
\end{array}\right) \\
& \in \mathbb{H} \cap \mathbb{M} .
\end{aligned}
$$

Therefore the system (7) is an interval observer for (2) with $\tilde{A}=\left(\begin{array}{ccc}-1.2464 & -0.0133 & -0.0182 \\ 0.4162 & -1.1899 & 0.8899 \\ 0.3002 & 0.5050 & -2.7636\end{array}\right)$,

$\tilde{C}=\left(\begin{array}{lll}0.7965 & 1.6303 & 2.2419\end{array}\right)$,

$\tilde{L}=\left[\begin{array}{lll}-0.0081 & 0.3969 & 0.1258\end{array}\right]^{T}$ and its initial conditions are selected as (8) where $\bar{x}(0)=\left[\begin{array}{lll}0.01 & 0.01 & 0.01\end{array}\right]^{T}$, $\underline{x}(0)=\left[\begin{array}{lll}-0.01 & -0.01 & -0.01\end{array}\right]^{T}$, satisfying the inclusion $\underline{x}_{0}(t) \leq x_{0}(t) \leq \bar{x}_{0}(t)$.

Under a sampling period $\mathrm{T}=0.01 \mathrm{~s},(11)$ and (12) can be computed with $\Phi=\left(\begin{array}{ccc}0.9875 & -0.0001 & -0.0002 \\ 0.0042 & 0.9881 & 0.0089 \\ 0.0030 & 0.0051 & 0.9724\end{array}\right)$, $\tilde{\tilde{L}_{1}}=\left[\begin{array}{lll}-0.0001 & 0.0040 & 0.0013\end{array}\right]^{T}$,

$\bar{\beta}=10^{-3}\left[\begin{array}{lll}0.1202 & 0.8019 & 0.3569\end{array}\right]^{T}, \quad \underline{\beta}=-\bar{\beta}$.

We plot in Fig. 5 both interval observer (10) and the predictor (13). We see that the interval observer exhibits the upper and lower bounds despite the uncertainties all the time while due to the network-induced delay $d$, the predictor provides two bounds of the state after a finite time $\bar{d}=2 s$. Hence, the theoretical developments are confirmed.
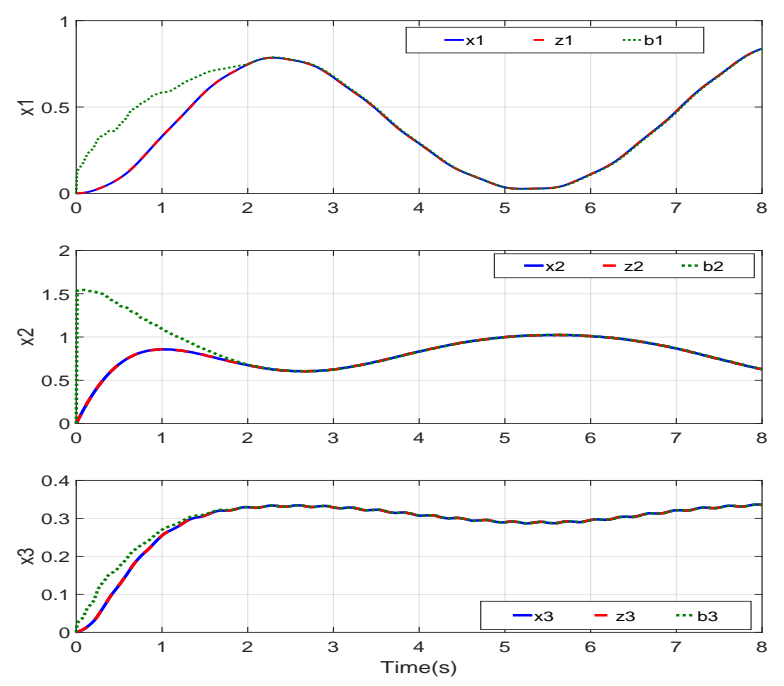

Fig. 4: Classical Approach.

\section{Conclusion}

In this paper, we have proposed a new technique of remote supervision for a class of NNCS with uncertainties (additive disturbances as well as measurement noises) and networkinduced delay. Based on Luenberger and interval observers, 

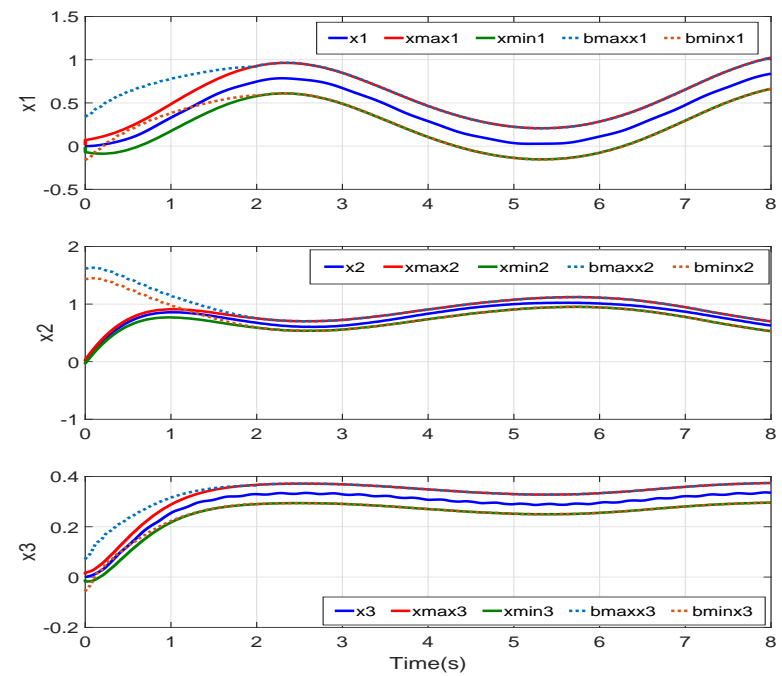

Fig. 5: Interval Approach.

two predictors are designed to provide, respectively, converging values of the solutions in the absence of uncertainties and a lower and an upper bounds when the uncertainties are present after a finite time which is the upper bound of the network-induced delay. The predictor-based controller for NNCS can be considered for future works.

\section{REFERENCES}

[1] X.-M. Zhang, Q.-L. Han, and X. Yu, "Survey on recent advances in networked control systems," IEEE Transactions on Industrial Informatics, vol. 12, no. 5, pp. 1740-1752, 2015.

[2] X. Ge, F. Yang, and Q.-L. Han, "Distributed networked control systems: A brief overview," Information Sciences, vol. 380, pp. 117131, 2017.

[3] D. Zhang, P. Shi, Q.-G. Wang, and L. Yu, "Analysis and synthesis of networked control systems: A survey of recent advances and challenges," ISA transactions, vol. 66, pp. 376-392, 2017.

[4] R. A. Gupta and M.-Y. Chow, "Overview of networked control systems," in Networked Control Systems. Springer, 2008, pp. 1-23.

[5] W. M. H. Heemels, A. R. Teel, N. Van de Wouw, and D. Nesic, "Networked control systems with communication constraints: Tradeoffs between transmission intervals, delays and performance," IEEE Transactions on Automatic control, vol. 55, no. 8, pp. 1781-1796, 2010.

[6] R. Yang, P. Shi, G.-P. Liu, and H. Gao, "Network-based feedback control for systems with mixed delays based on quantization and dropout compensation," Automatica, vol. 47, no. 12, pp. 2805-2809, 2011.

[7] C. Zhang, G. Feng, J. Qiu, and Y. Shen, "Control synthesis for a class of linear network-based systems with communication constraints," IEEE Transactions on Industrial Electronics, vol. 60, no. 8, pp. 33393348, 2013

[8] W.-A. Zhang and L. Yu, "Modelling and control of networked control systems with both network-induced delay and packet-dropout," Automatica, vol. 44, no. 12, pp. 3206-3210, 2008.

[9] M. Donkers, W. Heemels, N. Van de Wouw, and L. Hetel, "Stability analysis of networked control systems using a switched linear systems approach," IEEE Transactions on Automatic control, vol. 56, no. 9, pp. 2101-2115, 2011

[10] A. Kruszewski, W.-J. Jiang, E. Fridman, J. P. Richard, and A. Toguyeni, "A switched system approach to exponential stabilization through communication network," IEEE transactions on control systems technology, vol. 20, no. 4, pp. 887-900, 2011.
[11] Q. Zhu, K. Lu, and Y. Zhu, "Observer-based feedback control of networked control systems with delays and packet dropouts," Journal of Dynamic Systems, Measurement, and Control, vol. 138, no. 2, p. 021011,2016

[12] L. Zhang, Y. Shi, T. Chen, and B. Huang, "A new method for stabilization of networked control systems with random delays," IEEE Transactions on automatic control, vol. 50, no. 8, pp. 1177-1181, 2005.

[13] Y. Tang, H. Gao, and J. Kurths, "Robust $H_{\infty}$ self-triggered control of networked systems under packet dropouts," IEEE Transactions on cybernetics, vol. 46, no. 12, pp. 3294-3305, 2015.

[14] N. B. Am and E. Fridman, "Network-based $H_{\infty}$ filtering of parabolic systems," Automatica, vol. 50, no. 12, pp. 3139-3146, 2014.

[15] I. Pan, S. Das, and A. Gupta, "Tuning of an optimal fuzzy pid controller with stochastic algorithms for networked control systems with random time delay," ISA transactions, vol. 50, no. 1, pp. 28-36, 2011.

[16] L. Zhang, H. Gao, and O. Kaynak, "Network-induced constraints in networked control systemsa survey," IEEE transactions on industrial informatics, vol. 9, no. 1, pp. 403-416, 2013.

[17] S. Tong, S. Sui, and Y. Li, "Observed-based adaptive fuzzy tracking control for switched nonlinear systems with dead-zone," IEEE trans actions on cybernetics, vol. 45, no. 12, pp. 2816-2826, 2015.

[18] W. Jiang, C. Dong, E. Niu, and Q. Wang, "Observer-based robust fault detection filter design and optimization for networked control systems," Mathematical Problems in Engineering, vol. 2015, 2015.

[19] T. Dinh, F. Mazenc, and S.-I. Niculescu, "Interval observer composed of observers for nonlinear systems," in 13th European Control Conference, Strasbourg, France, 2014, pp. 660-665.

[20] T. Dinh and H. Ito, "Interval observers for continuous-time bilinear systems with discrete-time outputs," in 15th European Control Conference, Aalborg, Denmark, 2016, pp. 1418-1423.

[21] T. N. Dinh and H. Ito, "Decentralization of interval observers for robust controlling and monitoring a class of nonlinear systems," SICE Journal of Control, Measurement, and System Integration, vol. 10 no. 2, pp. 117-123, 2017.

[22] F. Mazenc and T. N. Dinh, "Construction of interval observers for continuous-time systems with discrete measurements," Automatica, vol. 50, no. 10, pp. 2555-2560, 2014.

[23] F. Mazenc, T. N. Dinh, and S.-I. Niculescu, "Interval observers for discrete-time systems," International Journal of Robust and Nonlinear Control, vol. 24, pp. 2867-2890, 2014.

[24] H. Zhang, Z. Zhang, Z. Wang, and Q. Shan, "New results on stability and stabilization of networked control systems with short time-varying delay," IEEE transactions on cybernetics, vol. 46, no. 12, pp. 2772 2781, 2015.

[25] L. Hetel, J. Daafouz, and C. Iung, "Analysis and control of lti and switched systems in digital loops via an event-based modelling," International Journal of Control, vol. 81, no. 7, pp. 1125-1138, 2008

[26] F. Mazenc and O. Bernard, "Interval observers for linear time-invarian systems with disturbances," Automatica, vol. 47, no. 1, pp. 140-147, 2011. 